\title{
La Poésie féminine française sera-t-elle enfin majeure en l'an 2000?
}

\author{
Annie Delpérier
}

\begin{abstract}
A border le thème de la poésie féminine contemporaine c'est se hasarder dans un paysage éclaté qui depuis le moyen-âge n'a constitué qu'une zone très marginale de la Poésie française. A l'exception des dictionnaires, ouvrages à intention exhaustives, ou des compilations spécifiques, la mémoire, telle qu'elle nous est restituée par les manuels scolaires et la majorité des anthologies que nous connaissons, n'aura pas installé dans son Panthéon plus d'une dizaine des femmes qui contribuèrent à l'élaboration de notre patrimoine intellectuel. Mais si Marie de France (fin du XIIe), Christine de Pisan (1364-1431), Pernette du Guillet (15201545) ou Marguerite d'Angoulême (1492-1549) sont très régulièrement citées parmi les poètes fondateurs de la littérature française, force est de constater, qu'elles ne le sont pas ensemble systématiquement.

Seuls quatre noms au fil des siècles se révèlent incontournables: Louise Labé (1524-1566), Marceline Desbordes-Valmore (1786-1859), Anna de Noailles (18761933) puis, plus près de nous, Marie Noèl (1883-1967). Encore, le statut de ces trois derniers poètes parait-il bien fragile comparé au génie rayonnant de la "Nymphe ardente du Rhône" dont l'Épître Dédicatoire de son oeuvre à Clémence de Bourges proclamait le féminisme intellectuel et social. Librement donc, somptueusement.
\end{abstract}

Louise Labé aura exprimé la violence des passions amoureuses, les émois de la chair, les exigences du coeur, avec une maîtrise telle, que toutes les attaques, favorisées par sa vie peut-être un brin dissolue, en restèrent sans effet.

A l'opposé, Marceline Desbordes-Valmore, célébrée par ses contemporains les plus prestigieux - Lamartine, Hugo, Vigny, Sainte-Beuve... - comme l'incarnation, l'essence même de son romantisme dont elle ouvrit les principales portes, se trouve aujourd'hui classée au rang des "romantiques-mineurs." Sans doute cette relégation peut-elle être attribuée paradoxalement au succès d'un mouvement dont les effets seraient reproduits jusqu'à la caricature, et qui mit longtemps en avant ses plèces les plus dérisoires. Que Marceline ait introduit avec la musicalité lyrique de son vers impair un genre dont s'inspireraient magistralement tour à tour, en lui en rendant grâce, Baudelaire et Verlaine, magicien de l'hendécasyllabe, ne change rien à l'affaire.

Belle, aristocrate, brillante, et terriblement parisienne, Anna de Noailles, née princesse Bibesco de Brancovan, puisa dans ses origines danubo-byzantines l'éclat et les subtilités d'une poésie traitant incomparablement de l'âme dans un pays qui depuis longtemps avait voué sa préférence à l'esprit; et c'est se référant non sans quelque malice intentionnelle à sa "sensualité potagère," que l'on met l'accent sur sa première période influencée par Barrès, panthéiste, et d'intense communion avec la nature. Son exceptionnel "romantisme féminin" - l'expression est de Charles Maurras - lui vaudra d'être toujours en 1983 l'unique femme poète du $\mathrm{XX}^{c}$ siècle figurant ès-qualité au Focus Bordas de la Littérature Française. 
Quant à la très provinciale Marie Noël, il est convenu de souligner qu'elle bénéficia surtout de la soudaine attention accordée à la poésie féminine de son temps pour venir au jour. De plus, sa spiritualité, sa compassion, l'humilité parfaite de son langage, l'apparentent trop au "Petit pauvre d'Assise" pour que l'on ne soit pas tenté de la présenter comme relevant davantage du ciel que de l'univers littéraire.

Tant de réserves sont-elle fondées? La participation des femmes, liée à leur évolution sociale, a-t-elle représenté seulement un apport non négligeable, certes, mais insuffisant à l'art poétique? ou bien ses caractéristiques s'entachent-elles des tares rédhtbitoires d'une "littérature du coeur," amplement décriée, et tout juste bonne à satisfaire la sentimentalité primaire d'un lectorat particulièrement inculte? A moins que "la cour des grands" de la Poésie française ne constitue une chasse gardée; un territoire fërocement masculin?

Le fait très banal de traiter à part aujourd'hui encore de la poésie fëminine justifie amplement ces interrogations en forme d'avertissement au lecteur qui nous accompagne.

Compte-tenu de l'isolement actuel du poète, conséquence de l'éparpillement Inéluctable auquel nous assistons depuis les deux ultimes et éphémères tentatives de regroupement nées de la guerre, qu'ont représenté La Poésie Résistante et L'École de Rochefort, toute approche du milieu poétique, quel qu'il soit, ne peut se prétendre qu'indicative, fragmentaire et profondément subjective.

Si l'on excepte de rares auteurs soutenus par les grandes maisons d'édition, la découverte d'authentiques talents s'apparente à un travail d'orpailleur; chance et patience lui sont indispensables. Un suivi attentif des prix poétiques importants, l'étude minutieuse du catalogue d'innombrables "petits éditeurs" intrépides où une plongée en immersion profonde dans l'inextricable maillage des centaines de revues francophones à tirage limité, - soutien vital de la Poésie actuelle - , nous livreront quelques-unes des rutilantes paillettes, ou inestimables pépites rêvées par notre convoitise. Ajoutons, qu'en dépit de l'image péjorative attachée à la démarche, le service de presse d'un tirage à compte d'auteur peut tout aussi bien constituer une révélation.

Mais avant de considérer certaines des composantes de la poésie de 1994 , accordons-nous de nous tourner vers les premières décennies de ce siècle pour en réveiller la voix exceptionnelle de la grande bergeracoise Catherine Pozzi (18821934). Fille du célèbre chirurgien Samuel Pozzi et épouse du dramaturge Edouard Bourdet, Catherine Pozzi, âme impérleuse, assoiffée d'unité, entretiendra au cours d'une vie brève et brillante, assombrie par la maladie, les drogues et la souffrance des dernières années, un ardent dialogue poétique et mystique avec Paul Valéry. Les oscillations de cette intimité bouleversante - uje vous ai forcé, ô dieu sauvage* lui écrivait-elle dans les premières exaltations de leur rencontre - Catherine Pozzi les livre au coeur de son Journal dont la parution chez Ramsay en 1987 a constitué un événement. Elle laisse un essail philosophique: Peau d'Ame et un recueil de poèmes - véritable perle noire - comptant, pour de nombreux spécialistes, parmi les plus admirables de notre langue: 


\section{Ave}

Très haut amour, s'il se peut que je meure

Sans avoir su d'où je vous possédais,

En quel soleil était votre demeure

En quel passé votre temps, en quelle heure

Je vous aimais;

Très haut amour quil passez la mémoire,

Feu sans foyer dont j'al fait tout mon jour,

En quel destin vous traciez mon histoire,

En quel sommeil se voyait votre gloire,

O mon séjour...

Quand je seral pour moi-même perdue

Et divisẻe à l'abîme infini.

Quand le présent dont je suis revêtue

Aura trahi.

Par l'univers en mille corps brisée,

De mille instants non rassemblés encor,

De cendre aux cieux jusqu'au néant vannée.

Vous referez pour une étrange année

Un seul trésor

Vous referez mon nom et mon image

De mille corps emportés par le jour,

Vive unité sans nom et sans visage.

Coeur de l'esprit, ô centre du mirage

Très haut amour.

C'est le beau visage d'Andrée Chedid, égyptienne d'origine libanaise, née au Caire, naturalisée française, qui domine sans conteste de sa solarité consensuelle la seconde moitié de ce siècle. Qu'apportait-elle donc de tellement unique, avec les couleurs miellées et les fragrances de son orient natal, la jeune femme venue en 1946 à la rencontre de Paris? Simplement, miraculeusement, l'équilibre, l'harmonie, la mesure et la fertilité de sa joie, Textes pour une figure (1949). A l'heure où l'Europe hébétée, tous repères abolis, émergeait d'une nuit effroyable, Andrée Chedid imposa le lyrisme lumineux de son verbe serein. Proclamant la pérennité de la vie, elle vint redonner son sens premier sacré à chaque manifestation du présent. Femme dépositatre d'un savoir ancestral que l'expérience alourdirait de ses drames, Textes pour le Vivant (1953) , elle sut recomposer l'enchainement des séquences de vie et de mort dans une juste acceptation d'un destin unitaire. «On ne peut bâtir qu'adossé à la mort, en même temps qu'accordé à demain," confierat-elle plus tard, livrant dans le poème "Sans Réponse" les raisons de sa force: 
A chaque oiseau qui sombre

Dans les marais de l'âme

A chaque souffle qui s'éteint

Dans le vaisseau du corps

J'interroge l'ingénieuse vie

Gardienne du chiffre et des arcanes

Sa réponse inaudible

Développe nos fictions

Féconde le terrain

Magnifie le parcours.

La fraternité, le bonheur, et les louanges de la beauté terrestre, Terre regardée (1957), s'offrirent en contrepoint au nihilisme triomphant et aux théories de l'absurde dont l'après-guerre foisonnait. Dans Prendre Corps, publiée en 1973, Andrée Chedid célèbre la Naissance et impose la femme, indifférente aux modes, dans les attributs de son règne: mère, centre de certitude, source d'amour et de rayonnement. Au cours d'un entretien accordé à Pierrette Micheloud pour le Journal des Poètes, en novembre 1993, elle confirmera d'ailleurs: "Il faut être soi-même, rien de plus, rien de moins, respecter sa propre voix intérieure, ne pas chercher à imiter ni à être original."

La guerre du Liban ouvrira en elle de cruelles blessures, et Cérémonial de la Violence (1976) succédera aux aspirations lointaines de Contre-Chant (1968) sans parvenir toutefois à réduire la confiance intuitive où s'enracine sa sagesse.

Toujours claire, attentive à la forme, préférant au charme trompeur de l'adjectif l'activité créatrice du verbe. Andrée Chedid a su se montrer maittresse, au travers de ses notes, conférences et entretiens, d'un discours poétique rigoureux et cohérent, au terme duquel: "La Poésie, comme tout art, détient un ferment continu, propose une cible heureusement inatteignable, un projet perfectible et jamais bouclé.*

Poète du vivant, Andrée Chedid affirme sans ambiguitté son refus de toute suggestion d'une vie post-mortem et son identification au présent. Interrogée sur l'espérance, elle répondit récemment: "Je me contente de vivre mon existence terrestre. Certains me sont étrangers." C'est une conception quil la différencle le plus radicalement, il me semble, de la majorité des poètes de cette fin de siècle, sombre de tous les effondrements qui le signent. En effet, la poésie féminine, globalement, semble converger vers la prophétie d'André Malraux: "Le troisième millénaire sera religieux ou ne sera pas." Aurait-il dit plutôt, ainsi que d'aucuns le prétendent: "sera spirituel ou ne sera pas" que la proposition conviendrait mieux encore, car si les pensées s'élèvent vers le Cosmos, interrogent l'au-delà, mysticisme, croyance et sens du religieux empruntent fréquemment des vecteurs ésotẻriques ou philosophiques étrangers aux religions instituées et à leurs interdits.

Le langage poétique d'Audrey Bernard fait surgir à nos yeux un monde toujours neuf, magnifié par la beauté de ses formulations. Ici, une mémoire ancienne inscrite au coeur des énergies guide la quête de "l'exacte lumière." Et il est vain de craindre, lorsque les éléments ordonnent autour de nous la symphonie qui nous tient dans son mystère essentiel. "Aqua / Fluorescence artérielle / Pour des oiseaux balises 
à remonter la mer..." L'éblouissement né d'un déluge d'images intensément colorées, où le verbe établit entre les figurants des rapports de communication inconnus, confèrent au poète le pouvoir sorcier d'ordonner, d'apaiser, de proposer son sens, au rythme du ressac des eaux dont elle s'instruit: "Patiente et animale / La mer est sur la plage / Comme une épiphanie en marche." La somptuosité, la fermeté et la constance de son écriture sont confirmées de recueil en recueil: Nuit des Hespérides, L'Éclat Dissident, Mémoire des Fleuves, Parfois la Mer, Vu du septlème Nuage, Pays sans visage.

La remontée des mémoires gouverne les voyages de Simonne Jacquemard à la recherche inlassable des mondes anciens où confluent nos histoires. Poète, romancière, ornithologue, danseuse et helléniste raffinée, elle a au cours de sa carrière publié plus de trente-quatre ouvrages, dont Le Vellleur de Nuit, aux éditions du Seuil, reçut en 1962 le prix Renaudot. Retirée à la campagne, au coeur de la réserve naturelle qu'elle a créée près de Sireuil, en Dordogne, elle enrichit de ses observations un savant bestiaire traversé d'un questionnement sans repos sur la vie. Sa poésie (Mers sans Rivages, 1945, Dérive au Zénith, 1965, Danse de l'Orée, 1979, La Bouche du Sphynx, 1992, Le Jardin d'Hérodote, 1994), haute, tendue, vibrante des ctvilisations millénaires, de terres dont elle s'imprègne avec une intensité médiumnique, nous tient dans le pouvoir brûlant d'une création venue des origines.

Née à Béziers, dans l'aire définie comme le Triangle d'Or de la poésie française, Antoinette Dard-Puech est l'héritière directe du vieux Sud où sur l'éternité du bûcher de Monségur flambe la pensée Cathare: "Actes et pensées / Sont comètes qui nous échappent / et s'en vont tracer dans l'êther / La route que nous devons prendre." Fière de son lignage elle s'est attachée à rendre vie aux Trobaïritz, ces célèbres femmes troubadours nées de la splendide civilisation occitane. Fille de la Méditerranée, Antoinette Dard-Puech en fait chatoyer les parures éternelles, la vigne, l'oltvier, le bleu du ciel, les ondulations lustrées de la mer afin que labandon contemplatif sertisse plus étroitement l'anxiété d'être: "O prisonnier / Quel ange nous habite?"

La poésie mystique de Jeanne Maillet de Saint-Pol-sur-Terrenoise (Les Palaces d'envergure, 1978, Ce parfum d'Immortelles, 1988. Chevaux-Légers d'une Histoire, 1994) éclate toutes les souffrances sublimées dans un chant de gloire et d'espérance nouvelle. La beauté et la force habitent ces vers en corollaires des certitudes dont lls naissent. Leur douceur nous atteint, leur quiétude nous impressionne. Et pour cette parole qui se fait passage, promesse, louange d'accompagnement, comment ne pas se prendre à rêver, entraînés par sillage d'un être déjà hors de portée, d'un parfum d'immortalité?

Une tranquillité semblable permet à Marie-Hélène Verdier (Le Prince du Lys, 1985, Bris de coeur, 1988) d'arpéger les raisons de sa fol en courtes visions aux colorations prismatiques devant lesquelles Pierre Emmanuel devait s'incliner en ces termes: "une intelligence angélique, une capacité d'émervelllement jamais en défaut devant la transparence de tout le créé."

Mais le langage de la quête métaphysique, cette tentation d'accéder à une vérité dépouillée, absolue, ne se construit pas aisément dans la luminosité qu'il appelle. La progression rigoureuse vers une perfection humaine de Cécile Zins selon les enseignements du bouddhisme zen: Adamah (Gallimard 1988), L'Arbre et la Glycine 
(1992) - intègre à son écriture altière, serrée sur sa logique, le mystère du kôan, histoire au sens délibérément obscur, destinée à faire surgir une réalité nouvelle de la déconstruction de concepts illusoires. Nous retrouvons une difficulté d'approche comparable, en dépit de sa beauté formelle, dans la poésie éthérée et fortement pensée de Catherine Guillery: Les Épines du Bien (1990), A ceux qui ont pris la terre (1994) - où s'éploie dans un ruissellement d'images et de relations symboliques la dualité du monde spirituel et sensible à la recherche de sa finalité.

A l'exemple de Claudine Helft (Métamorphose de l'Ombre, 1985, L'infint du Bleu, 1992), le lyrisme de Jeanne Benguigul, née en Algérie, emprunte largement à sa culture hébraïque. L'accueil réservé à Neuf Versets pour un Miracle, suivi de Corps dans la Demeure (Cahier Froissard 1991) commande que l'on s'arrête un instant sur l'expression tempêtueuse de cette âme tourmentée, écartelée entre le doute et la foi. Soif de pur Amour, hantise du Golgotha, appel de Vérité, s'expriment dans le déferlement d'un antique pathos méditerranéen à la mesure du Dieu qui l'inspire. La paix du corps aussi se conquiert au prix de souffrances exacerbées jusqu'à l'acceptation de cette "demeure sur la terre" qui se mue en Parole pour enfin se célébrer.

Un sentiment tragique de la vie donne à la volx d'Annie Salager - née en 1938 et professeur à Lyon, sa ville natale - la sonorité poignante des cordes. Discrète et sensible, elle égrène depuis Histoire pour le Jour (1968), les titres de recueils La femme-Buisson (1973), Les Fous de Bassan (1976). Figures du Temps sur une Eau Courante (1983), Chants (1988) - où dans les entrelacs d'un onirisme puissant la confidence se fait humaine, tissẻe d'amour et d'angoisse. Un rẻalisme assez rare pour être salué. comme l'aveu des désirs, des pulsions instinctives, la connaissance aiguẻ de la fugacité des moments ou de la douleur de la perte, nous retient au bord de cette écriture qui ne se trahit pas mais, tout au contraire, s'ennoblit en se chargeant des doutes et des faiblesses de l'être, pour mieux réaffirmer la valeur transcendante de l'amour universel. Le Tao a servi de base au chemin philosophique qu'Annie Salager tente de définir sous l'obscure densité du présent:

\section{Massacre}

On voudrait presser la lumière du jour, qu'elle puisse couler lentement comme un jardin dans un puits. On voudrait que la mémoire s'immobilise au coeur d'une rose en ressemblant à n'importe quelle abeille de rien du tout, on voudrait, mais elle se nourrit plus difficilement des sucs de lumière qu'elle ne s'empêtre dans un terreau meurtrl. En réalité, 11 pleut partout la barbarie, du dehors au dedans ça déchire. Et quand il ne reste rien du jour que la poussière, ça veut détruire encore ce peu de fumée qui s'élève des cendres en arrachant des cris.

Autre perception navrée en filigrane chez Colette Klein, poète et nouvelliste, née à Paris, quil, depuis son premier recueil (Ailleurs l'Étoile, 1973), ne cesse de captiver par la grâce d'un verbe désincarné qu'une minutieuse ciselure rend toujours plus limpide. Cette poésie, attentive à restituer tout son poids au silence, sait dire dans 
un murmure des lèvres, une vision anxieuse du monde. De nombreux recueils éclairent ce jugement: A Défaut de visage (1975), Cécités (1978), Le Passe Nuit (1980). Néante aux Mains d'Otseaux (1984), et Les Hautes Volières du Silence (1994).

L'intérêt de la poésie féminine contemporaine c'est sa cohérence et sa solidité. En dépit de la diversité des styles, une même adhésion mesurée à la recherche d'un langage moderne, où l'expression plus élaboree se concentre. La même prise en charge du fardeau de notre connaissance: le dérisoire de nos actions au sein d'un monde déchiré que le néant aveugle de toute part. Mais plus affirmée que l'accablement, la volonté farouche de trouver une vole, d'interroger plus ardemment l'univers, de reconquérir les sources essentielles en magnifiant une création avec laquelle l'être humain a perdu un accord naturel.

L'exemple de la solidité, de la fermeté d'une réflexion harmonisée au regard, à l'amour, au souvenir, à l'émervellement, mais encore à la structure, comme à la précision du langage, c'est dans l'oeuvre de Francine Caron, professeur agrégé de Lettres à l'Université de Rennes, que nous le retrouvons. Admirable assise pour un poète attaché à détailler et comprendre dans l'audace sensuelle et la justesse de la distanciation (Femmes Majeures, L'Année d'Amour, Picardie-Poésie, Musée du Louvre, Terres Celtes, Bretagne au Coeur, Grandeur nature, L'Amour le Feu).

Laisser croire que la poésie féminine s'est globalement détournée des problèmes concrets serait une imposture. Ce sont eux qui alimentent le grand fonds très vivace d'une poésie populaire, fortement irriguée par les courants classiques du XIX siècle. De même, c'est le plus souvent l'expérience du quotidien, l'anecdote, qui a inspiré à Micheline Dupray, née en 1927, depuis son premier recueil Au Clou des Ans, jusqu'à Crier l'absence (1989) une oeuvre tendre et sédulsante à la musicalité très prononcée.

La prise en compte des tragédies et menaces liées à notre époque a mis en exergue de par le monde une poésie d'engagement à laquelle des auteurs d'envergure ont lié leur plume. Rejoignant le combat pour l'écologie des poètes anglo-saxons, nous ne pouvons ignorer la Limousine Marcelle Delpastre, rendue célèbre par ses récits de vie: Les Chemins Creux (1993), Derrière les Murs (1994). Occitaniste en terre des troubadours, marcelle Delpastre s'impose par la puissance singulière d'une poésie lyrique hors du temps, libre d'attache, où roulent sous le regard d'un dieu lointain les thèmes éternels à notre condition. L'inspiration, le souffle et l'ampleur qui la portent ont attiré sur son oeuvre en langue française: L'Histoire dérisoire sutvie de Marguerite dans le Miroir et la Fulle Assise (1990) - attention et controverses passionnées. Inclassable Marcelle Delpastre? C'est possible; mais la force somptueuse et poignante de son cantique pour la terre: "Nous n'avons pas aimé assez, pour mériter la terre... ni la pensée qui veut et qui dépasse la pensée. Nous n'avons pas aimé nos rêves..." - La Terre (Traces 1970) - la désigne comme l'un des premiers poètes de ce pays qui ait jeté un regard responsable et lucide sur l'agonie de la planète.

Les grands courants de liberté qui ont fait frissonner l'histoire contemporaine marquent par paliers l'oeuvre de Denise Miège, née à Paris, journaliste, nouvelliste et auteur radiophonique. Une anthologie de littérature érotique fẻminine en deux volumes (1970 et 1973), un recueil d'érotologie arabe et une étude sur le ballet moderne ont accompagné la publication de cinq recueils de poèmes. Sous les Pavés 
la Plage (1969) restituait l'esprit effervescent de Mai 68 alors que les prtvilèges et les jeux désinvoltes de la liberté amoureuse récemment conquise trouvaient leurs échos dans La Mourre (1983).

Traditionnellement attachées à la féminité de l'écriture, les séductions que sont la musicalité, les capacités d'enchantement, l'art de traiter avec une tendresse légère des attachements et des nostalgies, de lincomparable préviosité des moments qui se savourent, ont fait place, nous le constatons, à une beauté plus grave. Elles continuent d'animer cependant avec une particulière élégance la poésie mésangère de Nadine Najman - Parcours Fléché (1987) - poète à facettes multiples, que l'on est tenté de comparer à ces maitres délicats de la poésie fantaisiste que furent Philippe Chabanneix et Marcel Beguey.

L'expression spontanée des sentiments éternels que nous nommons tendresse. amitié, révolte, jole, court dans la jolie poésie moderne et réfléchie de Danièle Labatsuzan (Des Ronds dans l'Eau, Sur DeuxMots, 1988). Les revues francophones sensibles à la fraîcheur inventive d'un langage profondément poétique ont accueilli en nombre cette "filleule" de Pierre Seghers: elle participa à sa première émission radiophonique auprès de lui à lâge de seize ans. Interprétée par Christian Ray la poésie chantée de Danièle Labatsuzan se révèle particulièrement adaptée aux nouvelles formes de diffusions offertes par la technique.

Enfin la création des courts poèmes polis de blanche nostalgie par Claudia Adrover - Lignage (1994) - répond exemplairement à cette urgence intérieure et vitale par laquelle tout art nait d'une reconstruction parallèle de l'être. La permanence de la Poésie est inscrite dans la paix de cet accomplissement. Royaume en Exil, La vie en marge, Le Moi Dormant, D'une Mer à l'Autre, Champs d'exil, Les Derniers Vents Doux, sont les autres titres des recueils de Claudia Adrover, née en Algérie et professeur de littérature anglo-américaine.

Les poèmes frémissants de sève sous la plume de Patricia Bruneaux ou encore ceux de Christine Keller, Andrée Marik, Evelyne Morin, Annie Le Gall, Katherine Bankhead, Eliane Demazet, Isabelle Poncet-Rimaud auratent pu tout aussi bien servir à illustrer mon parcours. Certains d'entre eux portent les promesses du futur. Beaucoup d'autres auxquels je ne me suis pas référée constituent la richesse d'aujourd'hui. Mais c'est grâce à des volx et des visages de femmes connues ou ignorées, attendues ou surprenantes que je tenais à témoigner de la réalité d'une poésie féminine française intelligente, assurée, féconde, et accordée aux réalités de notre temps. Une poésie abondamment représentée en revues et silencieusement ostracisée au-delà. L'heure n'est-elle point venue de se demander si l'an 2000 sera pour elle celui de la majorité? Celui où elle accédera au droit élémentaire de perdurer en Poésie, tout simplement.

Note

${ }^{1}$ Directrice, La Toison d'or, à Bergerac. 